\title{
ONE APPROACH FOR TRANSITIONING THE INET STANDARDS INTO THE IRIG 106 TELEMETRY STANDARDS
}

\author{
Carl Reinwald \\ Senior Principal Engineer \\ $\mathrm{CSC}^{\circledR}$, Santa Maria, California \\ Prepared by CSC for JT3, \\ Contract F42650-01-C-7218
}

\begin{abstract}
This paper documents one proposed approach for transitioning the Integrated Network Enhanced Telemetry (iNET) Standards into the IRIG 106 Part 2 Telemetry Network Standards. Describing the iNET Standards in terms of the Transmission Control Protocol/Internet Protocol (TCP/IP) Model provides a solid foundation for the proposed IRIG 106 Part 2 chapter structure. The proposed approach incorporates an application-centric paradigm by emphasizing application-toapplication communication. One change proposal augments the current Simple Network Management Protocol (SNMP) application protocol with a Hypertext Transfer Protocol (HTTP) application protocol that enables advanced data transfer features and other options. Another change proposal includes Data Channel enhancements resulting in a more harmonious relationship between statically and dynamically-defined Data Channels. This paper includes a high level transition plan for migrating towards this proposed approach for IRIG 106 Part 2.
\end{abstract}

\section{INTRODUCTION}

The Central Test and Evaluation Investment Program (CTEIP) launched the iNET project to help develop capabilities to support the increasing needs for test and evaluation of modern and upcoming military vehicles and systems [1]. By augmenting traditional point-to-point telemetry links with Internet Protocol (IP) networks, flight test capabilities could be greatly enhanced. A key iNET project objective was to develop a set of well-established and validated interoperability standards (referred to as the iNET Standards) with the ultimate goal of incorporating those standards into the Range Commander's Council (RCC) IRIG 106 Telemetry Standards [1]. In 2013, the RCC Telemetry Group (TG) initiated a task to review the iNET Standards and formulate an approach for transitioning the iNET Standards into the IRIG 106 Part 2 Telemetry Network Standards. This paper documents one proposed approach for the transition. Furthermore, this paper identifies several change proposals that the RCC TG may take under consideration for inclusion into IRIG 106 Part 2. Familiarity with the iNET Standards is assumed; for this proposed approach to IRIG 106 Part 2, the iNET abbreviation "TmNS" has been repurposed from "Telemetry Network System" to "Telemetry Network Standards". 


\section{INET STANDARDS LAYERED ON TCP/IP MODEL}

The iNET Standards consist of four documents: Test Article (TA) Standard [2], System Management (SM) Standard [3], Metadata Standard [4], and Radio Access Network (RAN) Standard [5]. These standards reference many existing protocols, most of which are part of the TCP/IP Protocol Suite. Figure 1 illustrates the Open Systems Interconnection (OSI) Model, the corresponding TCP/IP Model, and the major components of the TCP/IP Protocol Suite. Figure 2 represents the iNET-specific protocols layered onto the TCP/IP Model.

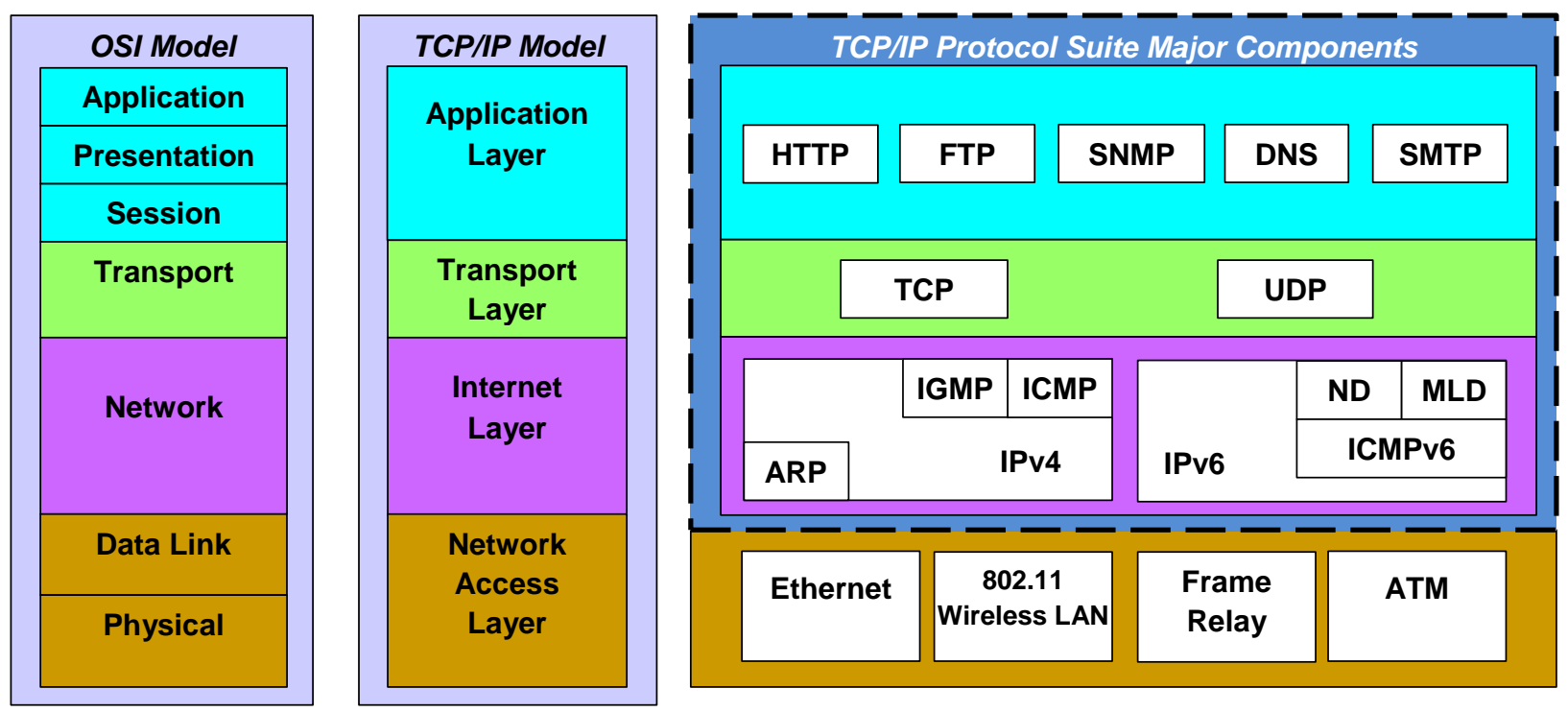

Figure 1. OSI and TCP/IP Model with TCP/IP Protocol Suite

\begin{tabular}{|c|c|c|c|}
\hline Application & Manager / Agent Apps & Data Transfer Apps & Radio Access Net Apps \\
\hline \multirow{4}{*}{$\begin{array}{l}\text { Application } \\
\text { Layer }\end{array}$} & & LTC Delivery Protocol & RF Net Mgmt Protocols \\
\hline & & RC Delivery Protocol & Link Mgmt Protocols \\
\hline & MDL Instance Doc & TmNS Messages & RAN Messages \\
\hline & iNET SM Protocols & iNET SM Protocols & iNET SM Protocols \\
\hline $\begin{array}{l}\text { Transport } \\
\text { Layer }\end{array}$ & \multicolumn{3}{|c|}{ No iNET-specific Transport Layer Protocols } \\
\hline $\begin{array}{c}\text { Internet } \\
\text { Layer }\end{array}$ & \multicolumn{3}{|c|}{ No iNET-specific Internet Layer Protocols } \\
\hline \multirow{2}{*}{$\begin{array}{c}\text { Network } \\
\text { Access } \\
\text { Layer }\end{array}$} & Link Layer & MAC Frames & ARQ Blocks \\
\hline & RF Physical Layer & SOQPSK-TG & LDPC Code Blocks \\
\hline
\end{tabular}

Figure 2. iNET-specific Protocols Layered onto the TCP/IP Model 


\section{INET STANDARDS OVERVIEW}

The iNET System Management Protocols (iNET SM Protocols) are implemented by all TmNS Applications; SNMP is the primary application protocol. The iNET SM Protocols include a Configuration Protocol for configuring an application using a Metadata Language (MDL) Instance Document. Data transfer applications use the Latency/Throughput Critical (LTC) and Reliability Critical (RC) Delivery Protocols to exchange measurement data in the form of Metadata-defined TmNS Data Messages. The Radio Frequency (RF) Network Management applications use RF Network and Link Management Protocols to control the transmission of data across the shared RF link. The iNET Standards leverage the existing TCP/IP Model's Transport and Internet Layer Protocols so the messages passed between the Application Layer protocols and the Network Access Layer are standard TCP segments and User Datagram Protocol (UDP) datagrams encapsulated in IP datagrams. The RF Link and Physical Layers transport IP datagrams across the RF link. The RF Link Layer incorporates a Time-Division Multiple Access channel access method for sharing RF spectrum between multiple participants. The RF Physical Layer modulation uses an RCC-TG defined modified version of the MIL-STD-188-181 Singlecarrier Offset Quadrature Phase Shift Keying, abbreviated as SOQPSK-TG.

From a high level perspective, the iNET Standards may be partitioned into the Radio Access Network (RAN) Standard and a set of application standards. The RAN Standard encompasses the RF Physical and Link Layers as well as all applications required to manage the shared RF spectrum. The RAN Standard provides the infrastructure for managing the IP wireless networks between Test Article applications and Ground applications. The application standards include standards for application configuration, control, and status; standards for measurement data transfer between applications; and standards for describing application configurations and the structure of application data messages. The application standards define the interoperability between applications communicating via the IP network. The RAN Standard provides the IP interconnectivity but without the application standards, there would be little vendor-independent interoperability between Test Article applications and Ground applications. Driven by the critical relationship between application standards and application interoperability, the proposed approach to IRIG 106 Part 2 focuses on standardizing application-to-application communication.

\section{PROPOSED IRIG 106 PART 2 CHAPTER STRUCTURE}

The IRIG Standard 106-13 Part 1, Telemetry Standards, contain Chapters $1-10$ and a set of appendixes labeled Appendix A, B, etc. The RCC-TG initially defined a second part to IRIG 106 named "IRIG Standard 106-xx Part 2, Telemetry Network Standards". To avoid any potential ambiguity with the existing IRIG 106 Part 1 chapters, the proposed approach is to start IRIG 106 Part 2 with Chapter 21 and have the appendixes labeled Appendix AA, BB, etc. With this chapter structure, the RCC-TG has the option of restoring IRIG 106 to a single Telemetry Standard where the chapters above Chapter 20 are part of the Telemetry Network Standards section (this is an option for the RCC-TG to consider). The RCC-TG was presented with the following proposed refactoring of the iNET Standards to consider for IRIG 106 Part 2: 
1. Split the TA Standard into three chapters:

a. Core Protocol Suite Chapter: identifies all of the core protocols available for use by TmNS Network Nodes.

b. Application Messages Chapter: defines application level messages and includes the TmNS Message structure definition.

c. Application Data Transfer Chapter: includes the existing LTC and RC Delivery Protocols and includes a change proposal to define an HTTP-based Data Channel protocol for transferring TmNS Messages between applications.

2. Abstract the SNMP Management Information Base (MIB) variables as Application Resources. The majority of the System Management Standard is included in the Application Resources Chapter.

3. Include only top-level descriptive information in the Metadata Chapter; the MDL schema already includes the explicit element definitions.

4. Split the RAN Standard in two chapters:

a. RF Network Access Layer Chapter: defines the RF Link and Physical Layers.

b. Radio Access Network Management Chapter: defines the Application Layer protocols for controlling and monitoring the RAN.

5. Include the MDL Extensible Markup Language (XML) Schema and TmNS MIB documentation in Appendixes.

6. Propose creation of TmNS Engineers Handbooks to capture Metadata Language Best Practices, RAN Supplemental Information, Quality of Service Management, and Application Development.

Figure 3 contains the proposed IRIG 106 Part 2 Telemetry Network Standards Table of Contents.

\begin{tabular}{c|l|l}
\multicolumn{1}{c}{ Chapter } & \multicolumn{1}{c}{ Chapter Title } & iNET Standard Document \\
\hline 21 & Introduction & None \\
\hline 22 & Core Protocol Suite & Test Article Standard \\
\hline 23 & Metadata & Metadata Standard \\
\hline 24 & Application Messages & Test Article Standard \\
\hline 25 & Application Resources & System Management Standard \\
\hline 26 & Application Data Transfer & Test Article Standard \\
\hline 27 & RF Network Access Layer & Radio Access Network Standard \\
\hline 28 & Radio Access Network Management & Radio Access Network Standard \\
\hline AA & $\begin{array}{l}\text { Metadata Language (MDL) XML Schema } \\
\text { Documentation }\end{array}$ & Metadata Standard \\
\hline BB & $\begin{array}{l}\text { Application Resources Language (ARL) } \\
\text { XML Schema Documentation }\end{array}$ & None \\
\hline CC & $\begin{array}{l}\text { Instrumentation Hardware Abstraction } \\
\text { Language (IHAL) XML Schema } \\
\text { Documentation }\end{array}$ & $\begin{array}{l}\text { None (IHAL XML schema already } \\
\text { defined in IRIG 106 Part 1, Chapter 9) }\end{array}$ \\
\hline DD & $\begin{array}{l}\text { TmNS Management Information Base } \\
\text { (TMNS-MIB) Documentation }\end{array}$ & System Management Standard \\
\hline
\end{tabular}

Figure 3. Proposed IRIG 106 Part 2 Telemetry Network Standards Table of Contents 
Figure 4 represents the proposed IRIG 106 Part 2-specific protocols layered onto the TCP/IP Model along with the proposed IRIG 106 Part 2 chapter references.

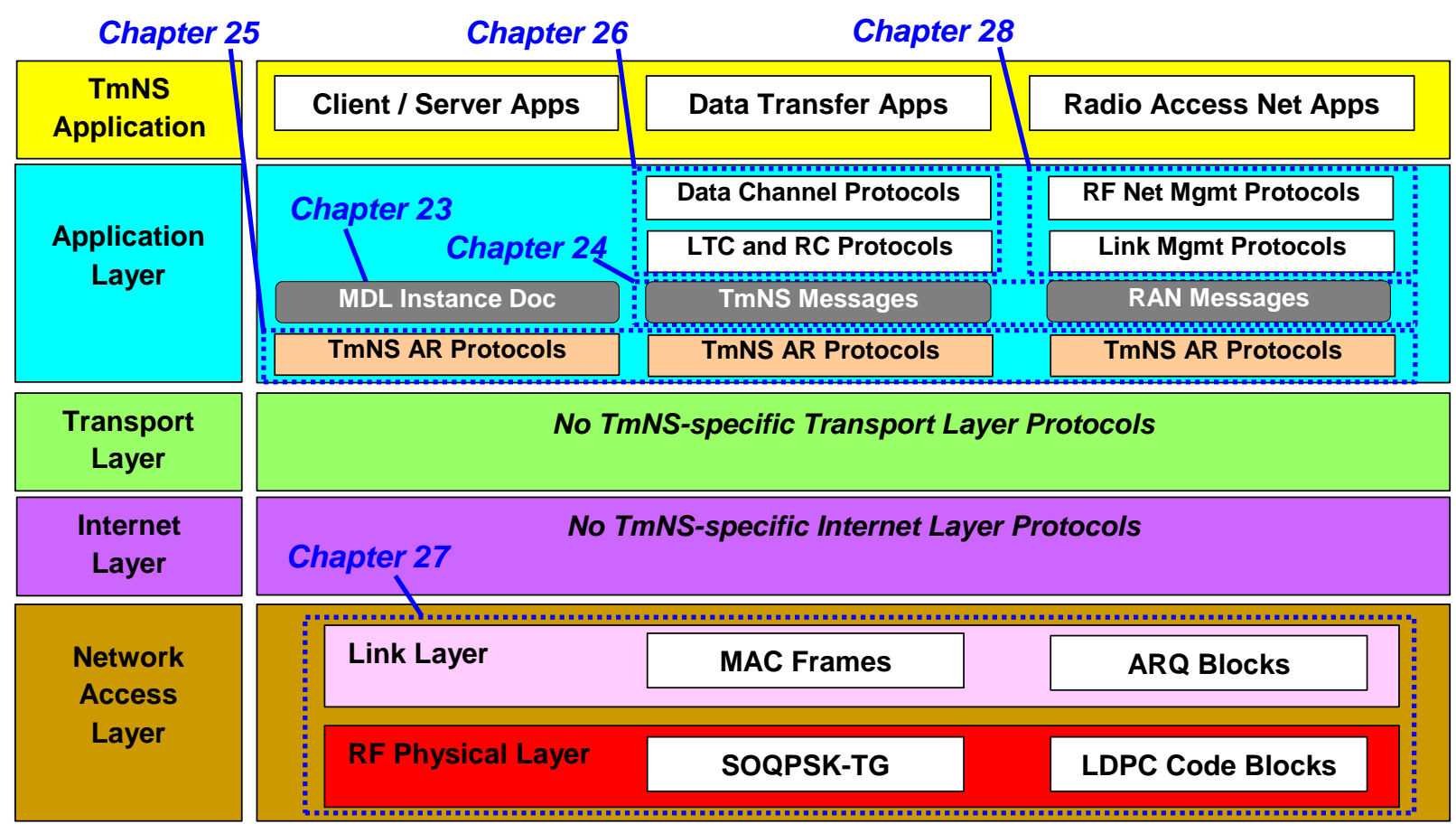

Figure 4. Proposed IRIG 106 Part 2-specific Protocols Layered onto the TCP/IP Model

\section{APPLICATION RESOURCES}

Application-to-application communication is an integral part of the iNET Standards. Each application implements an SNMP Agent with a set of common and application-specific MIB variables. The proposed approach to IRIG 106 Part 2 abstracts these MIB variables along with other functions into a set of application resources. Application communication occurs when one application accesses another application's resources. Many SNMP resources (i.e., MIB variables) implement specific functions (e.g., Data Source Transmit Enable) while some SNMP resources define a communication protocol (e.g., Configuration Protocol). SNMP resources are sufficient for simple control functions, but may not be the optimal application protocol for more complex application-to-application communication requirements.

One proposed addition to IRIG 106 Part 2 enhances the iNET System Management philosophy by augmenting the SNMP resources with HTTP resources. As compared to SNMP, HTTP coupled with XML technology provides the foundation for more robust and extensible communication protocols. The iNET Standards SNMP resources along with HTTP resources (and possibly other non-SNMP resources) could be placed under a single all-encompassing TmNS Application Resources Hierarchy. This hierarchy identifies each application resource and the applicable application layer protocol. A possible addition to this hierarchy would be the Instrumentation Hardware Abstract Language (IHAL) from IRIG 106 Part 1 Chapter 9. 
Figure 5 represents the top tier of one possible TmNS Application Resources Hierarchy; the RCC TG would ultimately be responsible for defining the hierarchy.

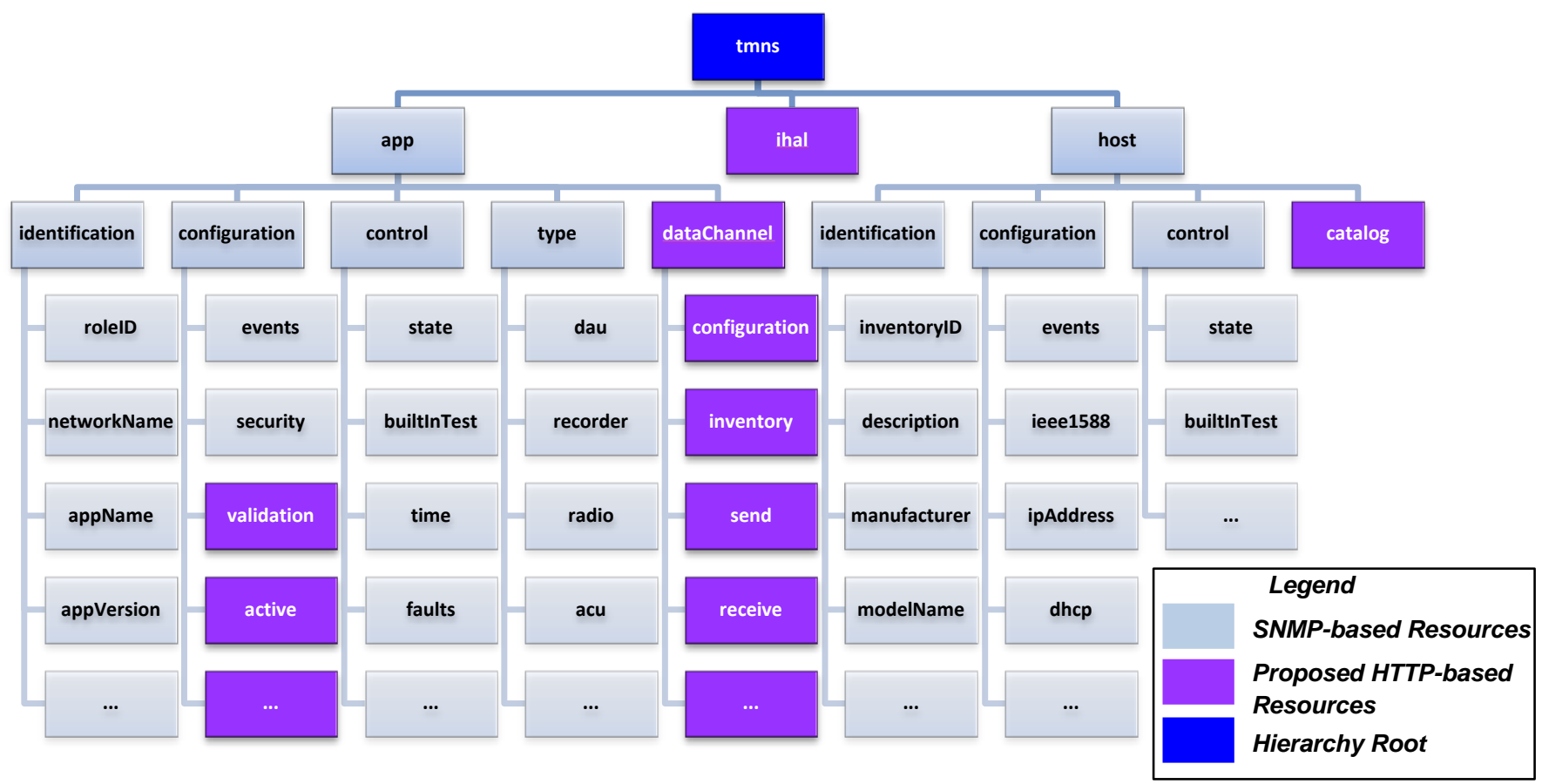

Figure 5. One Possible IRIG 106 Part 2 TmNS Application Resources Hierarchy

\section{INET DATA TRANSFER}

The iNET Standards define two delivery protocols: LTC and RC Delivery Protocols. Typically, LTC data transfers are instantiated via Metadata whereas RC data transfers are always instantiated via a client request to a data server. All Metadata-defined data transfers deliver data via UDP (over an LTC Data Channel) whereas client requests typically have data delivered via TCP. However, client requests may also deliver data via UDP (over an LTC Data Channel) so an LTC Data Channel may either be instantiated via Metadata or via a client request. Some consider the ambiguous origin of an LTC Data Channel a point of confusion within the iNET Standards. All LTC and RC data transfer statistics are reported via SNMP MIB variables.

All client requests are implemented using a customized version of the Real Time Streaming Protocol (RTSP), an HTTP-variant protocol designed for controlling streaming media servers. The iNET-customized RTSP requires an iNET-specific Uniform Resource Identifier (URI) (i.e., TmNS URI), an iNET-specific time format (i.e., PTP Time), and an independent TCP data channel connection protocol (RTSP's TCP delivery implementation interleaves binary data onto the TCP control channel). Once all of the iNET customizations are applied, only a small fraction of the original RTSP is actually used. 


\section{APPLICATION DATA TRANSFER}

A proposed adjustment to the iNET LTC and RC Delivery Protocols for IRIG 106 Part 2 is to define data transfer in the context of instantiating and maintaining Data Channels. A Data Channel identifies a logical network connection used to transfer TmNS data between a Data Source and Data Sink. A Data Channel is described by the following information:

- Network Transport Characteristics: includes destination address, port, transport protocol (UDP or TCP), direction (send or receive), and DiffServ Code Point level.

- Message List: nominally a list of Message Definition IDs.

- Time Range: comprised of a Start and End time.

Both Metadata and client request interfaces support the sending and receiving of data using either UDP or TCP. For Metadata-defined data transfers, a new Data Channel element structure is proposed to identify Message Definition IDs for transport along with a specific set of network transport parameters. While the iNET Standards restrict Metadata-defined data transfers to UDP, IRIG 106 Part 2 could support both UDP and TCP for Metadata-defined data transfers by including the proposed Data Channel approach.

Another proposed addition to IRIG 106 Part 2 involves client data requests. An HTTP-based data transfer interface could be added to IRIG 106 Part 2 that supports both sending and receiving of data using either UDP or TCP. Figure 6 shows the proposed request-based data transfer interface capabilities.

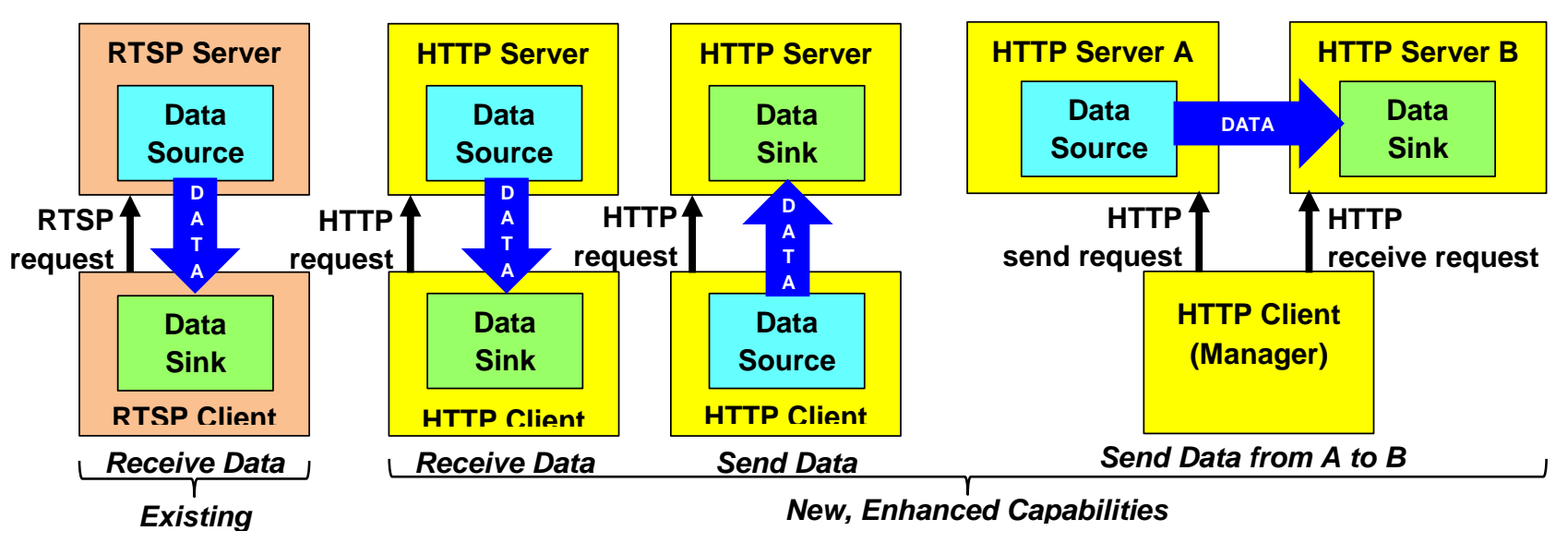

Figure 6. Proposed IRIG 106 Part 2 Request-based Data Transfer Interface Capabilities

The data transfer interface supports the retrieval of Data Channel information regardless of whether a Data Channel is instantiated via Metadata or via a client request. This highly extensible interface enables future extensions like data compression requests (e.g., send data currently sampled at 100 hertz only at 1 hertz) and data queries (e.g., return all samples outside a specific threshold range). If adopted, the instrumentation community could migrate away from the LTC and RC Delivery Protocols to the HTTP-based Data Channel approach. 


\section{TmNS MESSAGES}

The iNET Standards define the TmNS Data Message format for transporting measurement data. To facilitate future extensibility, another proposed change for IRIG 106 Part 2 generalizes this format into a TmNS Message by adding a Message Type field to the Message Header. A TmNS Message with a Message Type of zero would be functionally equivalent to the current iNET TmNS Data Message format (comparison shown in Figure 7). Other Message Types could be added to IRIG 106 Part 2 in the future.

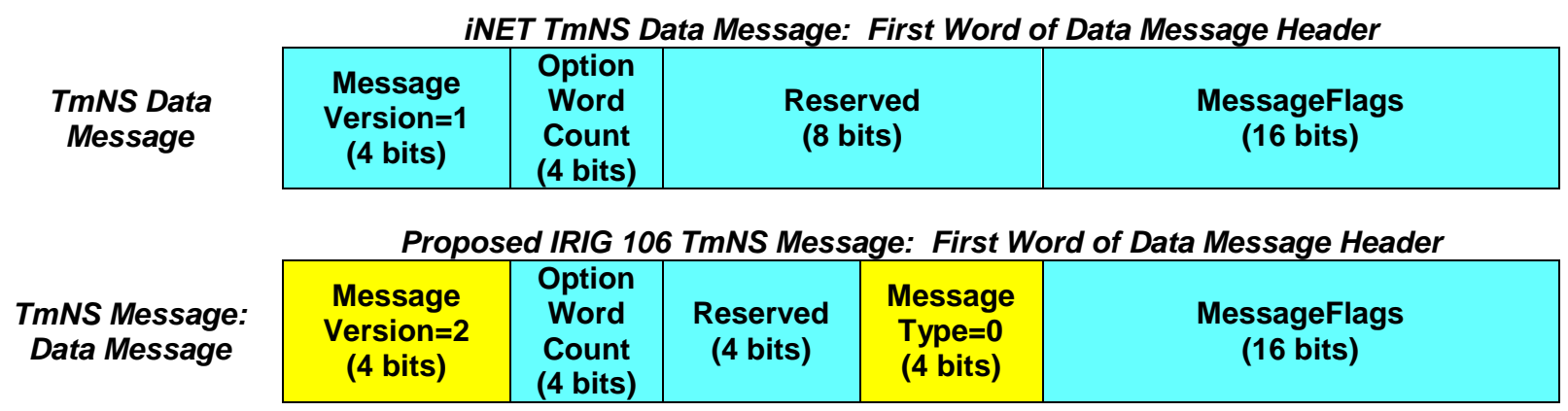

Figure 7. iNET TmNS Data Message Compared to Proposed IRIG 106 Part 2 TmNS Message Type 0

\section{TRANSITION PLAN}

The proposed transition plan for IRIG 106 Part 2 includes the chapter structure and the change proposals described previously. At this time, these proposed changes have not been approved by the RCC TG.

1. Change Proposals from iNET Standards to IRIG 106 Part 2:
a. Add Data Channel and associated elements to MDL.
b. Add Data Channel Protocol and HTTP-based data transfer interface.
c. Add TmNS Message Version 2.

2. Proposed Eventual Removal from IRIG 106 Part 2:
a. Remove network attributes from the MDL Message Definition element; replaced by Data Channel element (see 1a previously listed).
b. Remove LTC and RC elements from MDL (see 1a previously listed).
c. Remove LTC and RC MIB branches from the TmNS MIB; replaced by equivalent data statistics and control via HTTP-data transfer interface (see 1b previously listed).
d. Remove LTC and RC Delivery Protocols; replaced by Data Channel Protocol (see 1b previously listed).
e. Remove TmNS Message Version 1; replaced by Version 2 (see 1c previously listed). 
All items identified in \#1 above may be incorporated into IRIG 106 Part 2 without affecting existing iNET-compatible Network Nodes. The key to providing transitional support between the iNET Standards and the proposed IRIG 106 Part 2 lies in the Manager Applications that configure, control, and monitor TmNS Applications. With respect to MDL Instance Document generation, the current Manager Applications could implement items 1a above resulting in the generation of an MDL Instance Document compatible with existing iNET Network Nodes and IRIG 106 Network Nodes. For data channel monitoring, the Manager Applications could be enhanced to read Data Channel statistics via the HTTP-based Data Channel interface. Metadata could indicate whether a particular TmNS Application's Data Channel statistics are available via SNMP, HTTP, or both interfaces. Implementing Manager Applications that support both types of Network Nodes provides vendors with an elegant solution for the transitional coverage of their existing iNET Network Nodes while supporting new IRIG 106 Network Nodes.

Since the proposed approach to IRIG 106 Part 2 defines both TmNS Message Version 1 and Version 2, vendors could augment all Data Sinks to support both. For Data Sources, a vendor may choose to implement a Version 1 / Version 2 control to maintain compatibility with Version 1 Data Sinks. For the transition from the RTSP-based to HTTP-based data transfer protocol, Ground applications could be augmented with the capability of supporting both RTSP and HTTP concurrently. Although Test Article RTSP-based systems could also support both protocols concurrently, the limited Test Article hardware resources may make concurrent implementation impractical. As long as Ground applications support both protocols, the Test Article vendors may continue to support the RTSP-based interface. Figure 8 shows an example of the proposed dual standards Manager and Ground applications.
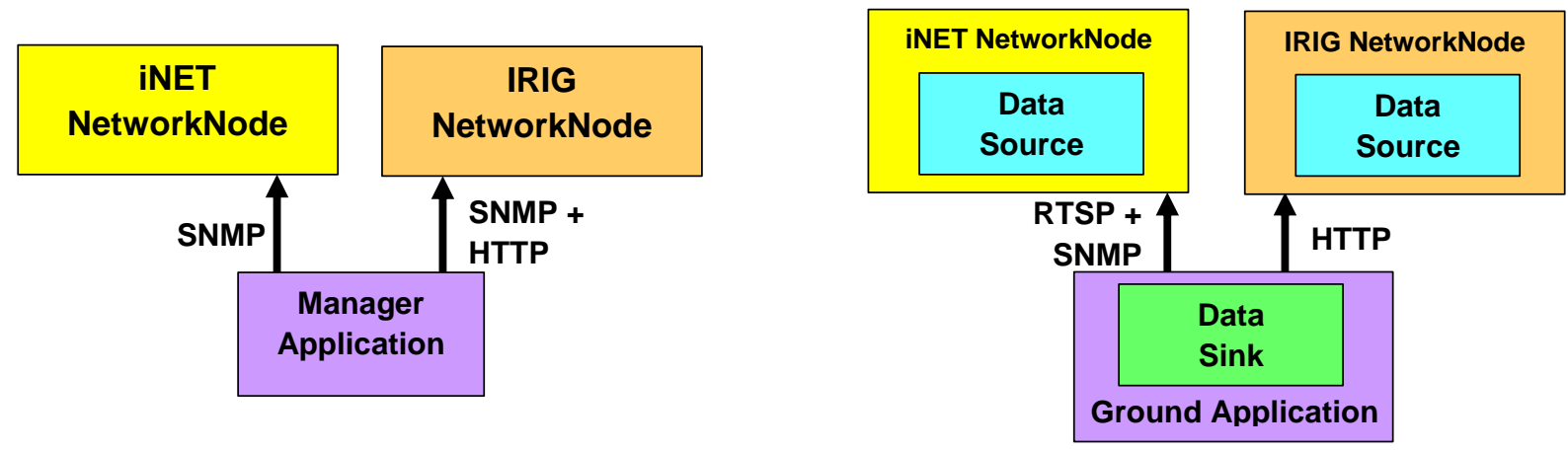

Figure 8. Example of Proposed Dual Standards Manager and Ground Applications

Once a smooth transition has been accomplished, items $2 \mathrm{a}-2 \mathrm{e}$ may eventually be removed from IRIG 106 Part 2 at the discretion of the RCC TG. 


\section{CONCLUSIONS}

Describing the iNET Standards in terms of the TCP/IP Model provides an excellent foundation for IRIG 106 Part 2. While preserving the iNET Standards' underlying requirements, the proposed approach for IRIG 106 Part 2 evolves the iNET Standards by implementing an application-centric paradigm. The proposed TmNS Application Resources Hierarchy encompasses all application level communication by including both SNMP and HTTP application protocols. The proposed Data Channel protocol unifies the concept of staticallydefined and dynamically-defined Data Channels. Thanks to the flexibility of the proposed enhancements, the transition plan for migrating from the iNET Standards to the proposed IRIG 106 Part 2 minimizes the overall impact of this transition on existing vendors' hardware. At this time, these proposed changes have not been approved by the RCC TG.

\section{ACKNOWLEDGEMENTS}

The author gratefully thanks CTEIP for supporting and funding the iNET project and the many members of the iNET Standards Working Groups for developing the iNET Standards. A special thanks to Bruce Lipe, Thomas Grace, Ray Faulstich, Jon Morgan, William Malatesta, Dave Robinson, the RCC TG Networks Committee, and the entire RCC TG Executive Committee for supporting this effort.

\section{REFERENCES}

[1] M. Moore, "Refined TmNS Architecture for the Integrated Network Enhanced Telemetry," Southwest Research Institute, San Antonio, TX, 2008.

[2] T. B. Grace, J. D. Kenney, M. L. Moodie and B. A. Abbott, "Key Components of the iNET Test Article Standard," in Processings of the International Telemetry Conference, Las Vegas, NV, 2009.

[3] T. B. Grace, A. R. Bertrand and T. A. Newton, "Applying the iNET System Management Standard," in Proceedings of the International Telemetry Conference, Las Vegas, NV, 2009.

[4] M. S. Moore, J. C. Price, A. R. Cormier and W. A. Malatesta, "Metadata Description Language: The iNET Metadata Standard Language," in Proceedings of the International Telemetry Conference, Las Vegas, NV, 2009.

[5] J. Kaba and B. Connolly, "Overview of the Telemetry Network System RF Data Link Layer," in Proceedings of the International Telemetry Conference, San Diego, CA, 2012. 